\title{
Composite M-branes
}

\author{
Miguel S. Costa* \\ D.A.M.T.P. \\ University of Cambridge \\ Silver Street \\ Cambridge CB3 9EW \\ England
}

September 1996

\begin{abstract}
We present new supersymmetric solutions of $D=11$ supergravity obtained by intersecting the brane configuration interpreted as a 2-brane lying within a 5-brane. Some of these solutions can be boosted along a common string and/or superposed with a Kałuża-Klein monopole. We also present a new embedding of the extreme four dimensional dyonic black hole with finite horizon area. These solutions are a consequence of a rather simple set of rules that allow us to construct the composite M-branes.
\end{abstract}

*email address: M.S.Costa@damtp.cam.ac.uk 


\section{Introduction}

Recently there has been a renewed interest in studying eleven dimensional supergravity brane solutions. This is due to a conjectured quantum theory in eleven dimensions, M-theory, whose effective field theory limit is $11 D$ supergravity [1, 2]. The latter has electric membrane [3] and magnetic 5-brane [4] solutions with charges arising from the 4-form field strength of this supergravity theory. These extreme solutions preserve half of the maximal supersymmetries and are believed to play a crucial role in a precise formulation of M-theory. In particular, the supermembrane admits a covariant Green-Schwartz action [5]. Double dimensional reduction of the supermembrane action from eleven to ten dimensions gives rise to the type IIA superstring action [6]. This is a strong evidence for some relation between an underlying quantum theory in eleven dimensions and type IIA superstring theory. The conjectured M-theory is believed to yield upon compactification the different superstring theories: compactification on $S^{1}$ gives rise to type IIA superstring theory [2]; the $E_{8} \times E_{8}$ heterotic string is believed to arrive in the reduction on $\frac{S^{1}}{Z_{2}}[7]$; the type II and the heterotic-type I dualities are related to the compactification of the M-theory on $T^{2}$ and on a $Z_{2}$ orbifold of $T^{2}$, respectively $[7-9]$.

It is known long ago that the basic electric 2-brane and magnetic 5-brane solutions of $11 D$ supergravity (now called M-branes) are not the only supersymmetric brane solutions of this theory [4. However, Townsend and Papadopoulos [10 have reinterpreted these other solutions as orthogonal intersections of M-branes and found other supersymmetric orthogonal intersecting solutions. Tseytlin [11] (see also [12, 13]) has extended this work and formulate a general rule, the 'harmonic function rule', to construct orthogonal intersecting branes with the basic 2 and 5 branes as its constituents. Some configurations can be boosted along a common string to all branes and/or superposed with a Kałuża-Klein (KK) monopole [14, 15] yielding, upon dimensional reduction to $D=10$, brane solutions with charges arising from the R-R 2-form field strength of type IIA superstring theory. There is however, another configuration of M-branes interpreted as a 2-brane lying within a 5 -brane $((2 \subset 5)$-brane $)$ corresponding to the uplift to eleven dimensions of the dyonic membrane of $N=2, D=8$ supergravity [16]. It seems therefore natural that there should be intersecting configurations with the previous solution among its constituents. The aim of this paper is to present new intersecting solutions of $(2 \subset 5)$-branes and to formulate general rules to construct the composite M-branes. Building all these M-brane configurations and deducing their composite rules is important not only to understand what M-theory may or may not be, but also to find new the supersymmetric brane solutions of string theories as these can be obtained from reductions of the composite M-branes and further duality transformations $[11,13,17-19]$. An interesting application is to perform the statistical counting of the entropy of extreme (or near extreme) black holes 
from an eleven dimensional perspective [12, $20-22]$.

This paper is organised as follows. In section two we set our notation and state the rules to construct the composite M-branes. In section three we present the new solutions: $(2 \subset 5) \perp(2 \subset 5) \perp(2 \subset 5),(2 \subset 5) \perp 5+$ boost,$(2 \subset 5) \perp$ $2+K K$ monopole, $(2 \subset 5)+$ boost $+K K$ monopole and $2 \perp 2 \perp 2+K K$ monopole branes. The latter is an embedding of the extreme four dimensional dyonic black hole with finite horizon area $[23-25]$. In section four we present a table with the composite M-brane scan and give some concluding remarks.

\section{Composite M-brane rules}

The action for the bosonic sector of eleven dimensional supergravity is

$$
I_{11}=\frac{1}{2} \int d^{11} x \sqrt{-g}\left[R-\frac{1}{2.4 !} \mathcal{F}^{2}\right]+\frac{1}{12} \int \mathcal{F} \wedge \mathcal{F} \wedge \mathcal{A},
$$

where $\mathcal{F}=d \mathcal{A}$ and $\mathcal{A}$ is a 3 -form field. The equations of motion that follow from this action are

$$
\begin{aligned}
& R_{M N}=\frac{1}{2.3 !}\left(\mathcal{F}_{M P Q R} \mathcal{F}_{N}{ }^{P Q R}-\frac{1}{12} g_{M N} \mathcal{F}^{2}\right), \\
& \partial_{M}\left(\sqrt{-g} \mathcal{F}^{M N P Q}\right)+\frac{1}{2.4 ! 4 !} \epsilon^{N P Q R_{1} \ldots R_{8}} \mathcal{F}_{R_{1} \ldots R_{4}} \mathcal{F}_{R_{5} \ldots R_{8}}=0,
\end{aligned}
$$

where $\epsilon$ is the alternating symbol defined by $\epsilon^{t x_{1} \ldots x_{10-p} y_{1} \ldots y_{p}}=1$ with $y_{i}$ the spatial coordinates of the anisotropic $p$-brane and $x_{i}$ the remaining spatial coordinates. A given bosonic background is said to be supersymmetric if there is a Killing spinor field solving the equation

$$
\delta \Psi_{M}=\left[D_{M}+\frac{1}{288}\left(\Gamma_{M}^{N P Q R}-8 \delta_{M}^{N} \Gamma^{P Q R}\right) \mathcal{F}_{N P Q R}\right] \epsilon=0,
$$

where we use the gamma matrices algebra $\left\{\Gamma_{A}, \Gamma_{B}\right\}=2 \eta_{A B}$. As usual $\Gamma_{M}=$ $e_{M}^{A} \Gamma_{A}$, where $M, N, \ldots$ denote world indices and $A, B, \ldots$ tangent space indices.]

Now we define the relative transverse space to a given $q_{i}$-brane. A $q_{1}$-brane intersecting a $q_{2}$-brane over a $r$-brane can be seen as an anisotropic $\left(p=q_{1}+\right.$ $\left.q_{2}-r\right)$-brane, and further on for configurations with more than two intersecting branes. The tangent vectors to the basic $q_{i}$-branes' worldvolume that are not tangent to the $r$-brane's worldvolume span the relative transverse space. The overall transverse space is spanned by the vectors orthogonal to the $r$-brane's worldvolume and to the relative transverse space [10]. The relative transverse space to a given $q_{i}$-brane, denoted by $\mathcal{M}_{(q, i)}^{p-q}$, is the space spanned by the vectors that are orthogonal to the $q_{i}$-brane's worldvolume and that are tangent to the anisotropic p-brane's worldvolume.[]

\footnotetext{
${ }^{1}$ We will order the world indices in $e_{M}^{A}$ according to $t y_{1} \ldots y_{p} x_{1} \ldots x_{10-p}$.

${ }^{2}$ If we have a configuration with the harmonic functions independent of $n$ directions of the
} 


\subsection{Orthogonal intersections of 2 and 5 branes}

The basic $\frac{1}{2}$ supersymmetric 2-brane solution of $11 D$ supergravity is described by $(2 \leq p \leq 7)$

$$
\begin{aligned}
& d s^{2}=H^{\frac{1}{3}}\left[H^{-1}\left(-d t^{2}+d y_{1}^{2}+d y_{2}^{2}\right)+d y_{3}^{2}+\ldots+d y_{p}^{2}+d x^{i} d x_{i}\right], \\
& * \mathcal{F}_{(2)}=Q\left(\epsilon_{9-p} \wedge \eta\right)
\end{aligned}
$$

where $H=1+\frac{\alpha}{r^{8-p}}$ is a harmonic function on the overall transverse space and $r^{2}=x^{i} x_{i}$ with $i=1, \ldots, 10-p . \quad \eta=d y_{3} \wedge \ldots \wedge d y_{p}$ is the volume form on the 2-brane relative transverse space $\mathcal{M}_{(2)}^{p-2}$ and $\epsilon_{9-p}$ the unit $(9-p)$-sphere volume form. The electric charge is defined by $Q=\frac{1}{V_{(2)}^{p-2}} \int_{\Sigma} * \mathcal{F}_{(2)}$, where $V_{(2)}^{p-2}$ is the volume of $\mathcal{M}_{(2)}^{p-2}$ and $\Sigma=S^{9-p} \times \mathcal{M}_{(2)}^{p-2}$ is an asymptotic spacelike hypersurface.[ The charge $Q$ is related to the positive constant $\alpha$ by

$$
\frac{Q}{(8-p) A_{9-p}}= \pm \alpha,
$$

where $A_{9-p}$ is the volume of the unit $(9-p)$-sphere.

This solution has a Killing spinor field given by

$$
\epsilon=H^{-\frac{1}{6}} \epsilon_{0}
$$

where $\Gamma_{012} \epsilon_{0}=\mp \epsilon_{0}$. The $\mp$ sign choice corresponds to positive or negative charge, respectively. This solution can be generalised to the corresponding multi-centered cases. In order to keep the notation less cumbersome we will consider just brane solutions centered at the origin.

The other $\frac{1}{2}$ supersymmetric basic solution is the magnetic 5 -brane described by $(5 \leq p \leq 7)$

$$
\begin{aligned}
& d s^{2}=H^{\frac{2}{3}}\left[H^{-1}\left(-d t^{2}+d y_{1}^{2}+\ldots+d y_{5}^{2}\right)+d y_{6}^{2}+\ldots+d y_{p}^{2}+d x^{i} d x_{i}\right], \\
& \mathcal{F}_{(5)}=P\left(\mu \wedge \epsilon_{9-p}\right)
\end{aligned}
$$

overall transverse space then these $n$ directions belong to the anisotropic $p$-brane worldvolume, e.g. a 2-brane will be seen as an anisotropic $(p=2+n)$-brane. These $n$ directions of the overall transverse space also belong to the branes' relative transverse spaces. The corresponding coordinates will be denoted by $y_{i}$.

${ }^{3}$ The Hodge dual of a $n$-form $\mathcal{F}$ in a $D$ dimensional manifold with Lorentzian signature is defined by $(* \mathcal{F})_{B_{1} \ldots B_{D-n}}=\frac{1}{n !} \sqrt{-g} \epsilon_{A_{1} \ldots A_{n} B_{1} \ldots B_{D-n}} \mathcal{F}^{A_{1} \ldots A_{n}}$ and satisfies $*^{2}=(-1)^{1+n(D-n)}$. We define $\epsilon_{t x_{1} \ldots x_{D-1-p} y_{1} \ldots y_{p}}=-1$ such that $\epsilon^{A_{1} \ldots A_{D}} \epsilon_{A_{1} \ldots A_{D}}=-D$ !. We will always define the contribution to $\mathcal{F}$ of an electric charged basic $(q=n-2)$-brane such that $\mathcal{F}_{(q)_{t r y_{i} \ldots y_{i+q}}}=$ $\frac{Q}{A_{D-2-p}} \frac{H^{-2}}{r^{D-2-p}}$ for some $i$. 
where $H=1+\frac{\alpha}{r^{8-p}}$ is a harmonic function on the overall transverse space, $r^{2}=x^{i} x_{i}$ with $i=1, \ldots, 10-p$ and $\mu=d y_{6} \wedge \ldots \wedge d y_{p}$ is the volume form on $\mathcal{M}_{(5)}^{p-5}$. The magnetic charge is defined by $P=\frac{1}{V_{(5)}^{p-5}} \int_{\Sigma} \mathcal{F}_{(5)}$, where $V_{(5)}^{p-5}$ is the volume of $\mathcal{M}_{(5)}^{p-5}$ and $\Sigma=\mathcal{M}_{(5)}^{p-5} \times S^{9-p}$. The charge $P$ is also given by the formula $(2.5)$.

The Killing spinor field is

$$
\epsilon=H^{-\frac{1}{12}} \epsilon_{0}
$$

where $\Gamma_{678910} \epsilon_{0}=\mp \epsilon_{0}$. The $\mp$ sign choice corresponds to positive or negative charge, respectively. 目

The previous basic branes can be used to construct supersymmetric configurations of $\mathrm{N}$ branes intersecting each other orthogonally [10, 11]. These configurations may be seen as anisotropic $p$-branes and can be built according to the following rules:

(i) To each basic $q_{i}$-brane we assign an harmonic function $H_{i}$ on the overall transverse space. If the coordinate $y$ belongs to several constituents $q_{i}$-branes $\left(q_{1}, \ldots, q_{n}\right)$ then its contribution to the metric written in the conformal frame where the overall transverse space is 'free' is $H_{1}^{-1} \ldots H_{n}^{-1} d y^{2}$ [11. . The contribution to the conformal factor of the i-th $q_{i}$-brane is $H_{i}^{\frac{q_{i}+1}{9}}$. The 4 -form field strength is given by

$$
\mathcal{F}=\sum_{i=1}^{N} \mathcal{F}_{(q, i)}
$$

where

$$
* \mathcal{F}_{(2, i)}=Q_{i}\left(\epsilon_{9-p} \wedge \eta_{i}\right), \quad \mathcal{F}_{(5, i)}=P_{i}\left(\mu_{i} \wedge \epsilon_{9-p}\right),
$$

whether the $\mathrm{i}$-th brane is a 2 or 5 -brane, respectively. In the former case, $\eta_{i}$ is the volume form on the i-th 2-brane relative transverse space $\mathcal{M}_{(2, i)}^{p-2}$ and the electric charge is defined by $Q_{i}=\frac{1}{V_{(2, i)}^{p-2}} \int_{\Sigma} * \mathcal{F}_{(2, i)}$ with $\Sigma=S^{9-p} \times \mathcal{M}_{(2, i)}^{p-2}$ and $V_{(2, i)}^{p-2}$ the volume of $\mathcal{M}_{(2, i)}^{p-2}$. In the latter case, $\mu_{i}$ is the volume form on $\mathcal{M}_{(5, i)}^{p-5}$ and the magnetic charge is defined by $P_{i}=\frac{1}{V_{(5, i)}^{p-5}} \int_{\Sigma} \mathcal{F}_{(5, i)}$ with $\Sigma=\mathcal{M}_{(5, i)}^{p-5} \times S^{9-p}$ and $V_{(5, i)}^{p-5}$ the volume of $\mathcal{M}_{(5, i)}^{p-5}$. Both the electric and magnetic charges are given by

$$
\frac{Q_{i}}{(8-p) A_{9-p}}, \frac{P_{i}}{(8-p) A_{9-p}}= \pm \alpha_{i} .
$$

\footnotetext{
${ }^{4}$ We order the gamma matrices indices such that they grow from the left to the right. This way there is no confusion between $\Gamma_{01}=\Gamma_{[0} \Gamma_{1]}$ and $\Gamma_{10}$. The latter is defined by $\Gamma_{10}=\Gamma^{10}=$ $\Gamma^{0} \Gamma^{1} \ldots \Gamma^{9}$.

${ }^{5}$ There is an extra minus sign if $y$ is the time coordinate $t$.
} 
(ii) $q$-branes of the same type can intersect orthogonally over $(q-2)$-branes [10]. A 2-brane can intersect orthogonally a 5-brane over a string [26, 27].

\subsection{Intersections with the $(2 \subset 5)$-brane}

Consider now the M-brane configuration interpreted as a 2-brane lying within a 5 -brane [16], i.e. the $(2 \subset 5)$-brane $(5 \leq p \leq 7)$

$$
\begin{aligned}
d s^{2}= & (H \tilde{H})^{\frac{1}{3}}\left[H^{-1}\left(-d t^{2}+d y_{1}^{2}+d y_{2}^{2}\right)\right. \\
& \left.+\tilde{H}^{-1}\left(d y_{3}^{2}+d y_{4}^{2}+d y_{5}^{2}\right)+d y_{6}^{2}+\ldots+d y_{p}^{2}+d x^{i} d x_{i}\right], \\
\mathcal{F}_{(2 \subset 5)}= & \mathcal{F}_{(2)}+\mathcal{F}_{(5)}-(8-p) \frac{\alpha \sin 2 \zeta}{2} \frac{\tilde{H}^{-2}}{r^{9-p}}(d r \wedge \xi),
\end{aligned}
$$

where $* \mathcal{F}_{(2)}=Q\left(\epsilon_{9-p} \wedge \eta\right), \mathcal{F}_{(5)}=P\left(\mu \wedge \epsilon_{9-p}\right), H=1+\frac{\alpha}{r^{8-p}}$ and $\tilde{H}=1+\frac{\tilde{\alpha}}{r^{8-p}}$ are harmonic functions on the overall transverse space, $r^{2}=x^{i} x_{i}$ with $i=1, \ldots, 10-p$ and $\tilde{\alpha}=\alpha \cos ^{2} \zeta$. If $\cos \zeta=0$ we obtain the 2-brane solution (2.4) and if $\sin \zeta=0$ the 5-brane solution (2.7). $\mu=d y_{6} \wedge \ldots \wedge d y_{p}$ and $\eta=d y_{3} \wedge d y_{4} \wedge d y_{5} \wedge \mu$ are the volume forms on the relative transverse spaces of the constituent M-branes $\left(\mathcal{M}_{(5)}^{p-5}\right.$ and $\left.\mathcal{M}_{(2)}^{p-2}\right) . \xi=d y_{3} \wedge d y_{4} \wedge d y_{5}$ is the volume form on the space $\mathcal{M}_{(5 / 2)}^{3}$ spanned by the vectors that are tangent to the 5-brane's worldvolume but are orthogonal to the 2-brane's worldvolume. The dual operation in the expression for $\mathcal{F}_{(2)}$ is defined for the metric with $\tilde{\alpha}=0$. The electric and magnetic charges are defined as for the previous basic solutions and they are given by 0

$$
\frac{Q}{(8-p) A_{9-p}}=\alpha \sin \zeta, \frac{P}{(8-p) A_{9-p}}=\alpha \cos \zeta .
$$

This background preserves half of the supersymmetries, admitting the following Killing spinor field

$$
\epsilon=(H \tilde{H})^{-\frac{1}{6}}\left[\left(\tilde{H}^{\frac{1}{2}} \pm H^{\frac{1}{2}} \cos \zeta\right)^{\frac{1}{2}}+\left(\tilde{H}^{\frac{1}{2}} \mp H^{\frac{1}{2}} \cos \zeta\right)^{\frac{1}{2}} \gamma\right] \epsilon_{0},
$$

where

$$
\Gamma_{012} \gamma \epsilon_{0}=\mp \epsilon_{0},
$$

with $\gamma=\Gamma_{012} \Gamma_{678910}$. The upper signs choice is for $\sin \zeta \geq 0$ and the lower signs choice for $\sin \zeta \leq 0$. If $\cos \zeta=0$ or $\sin \zeta=0$ we obtain Killing spinor fields that can be written as in (2.6) or (2.8), respectively.

\footnotetext{
${ }^{6}$ We remark that from the 4 -form field strength equation in (2.2) the conserved electric charge is in fact given by $Q=\frac{1}{V_{(2)}^{p-2}} \int_{\Sigma}\left(* \mathcal{F}+\frac{1}{2} \mathcal{A} \wedge \mathcal{F}\right)$. Even thought the Chern-Simons term does not vanish for this solution, we can always choose a gauge such that it vanishes at spatial infinity and therefore the electric charge may still be defined as before.
} 
Configurations of intersecting M-branes including the $(2 \subset 5)$-branes as possible constituents can be constructed according to the rule:

(iii) If there are $(2 \subset 5)$-branes among the $N$ intersecting constituents then in the cases where these branes reduce to the basic 2 or 5 -branes the resulting configuration should be compactible with the previous rules (i) and (ii). The 4-form field strength is given by

$$
\mathcal{F}=\sum_{i=1}^{N} \mathcal{F}_{(q, i)},
$$

where $\mathcal{F}_{(q, i)}$ is given either by one of the expressions in $(2.10)$ or by

$$
\mathcal{F}_{(2 \subset 5, i)}=\mathcal{F}_{(2, i)}+\mathcal{F}_{(5, i)}-(8-p) \frac{\alpha_{i} \sin 2 \zeta_{i}}{2} \frac{\tilde{H}_{i}^{-2}}{r^{9-p}}\left(d r \wedge \xi_{i}\right) .
$$

The dual operations are defined for $\tilde{\alpha}_{i}=0$ and $\xi_{i}$ is the volume form on the space $\mathcal{M}_{(5 / 2, i)}^{3}$. The electromagnetic charges are defined as before. They are given by

$$
\frac{Q_{i}}{(8-p) A_{9-p}}=\alpha_{i} \sin \zeta_{i}, \frac{P_{i}}{(8-p) A_{9-p}}=\alpha_{i} \cos \zeta_{i}
$$

where $\cos \zeta_{i}=0$ or $\sin \zeta_{i}=0$ if the $\mathrm{i}$-th brane is a basic 2 or 5 -brane, respectively.

\subsection{Kałuża-Klein charges}

In some configurations we can add KK charges yielding, upon dimensional reduction to $D=10$, branes with charges arising from the $\mathrm{R}$-R 2 -form field strength of type IIA superstring theory. The Kałuża-Klein reduction of $11 D$ supergravity yields $N=2 A, D=10$ supergravity. The corresponding bosonic sector action written in the string frame is given by

$$
\begin{aligned}
I_{I I A}= & \frac{1}{2} \int d^{10} x \sqrt{-g}\left[e^{-2 \phi}\left(R+4(\nabla \phi)^{2}-\frac{1}{2.3 !} \mathcal{H}^{2}\right)-\frac{1}{2.2 !} \mathcal{F}_{2}^{2}-\frac{1}{2.4 !} \mathcal{F}_{4}^{\prime 2}\right] \\
& +\frac{1}{4} \int \mathcal{F}_{4} \wedge \mathcal{F}_{4} \wedge \mathcal{B},
\end{aligned}
$$

where $\mathcal{F}_{4}^{\prime}=d \mathcal{A}_{3}+\mathcal{A} \wedge \mathcal{H}, \mathcal{F}_{4}=d \mathcal{A}_{3}, \mathcal{H}=d \mathcal{B}, \mathcal{F}_{2}=d \mathcal{A}$ and $\mathcal{A}, \mathcal{B}$ and $\mathcal{A}_{3}$ are 1 , 2 and 3 -form fields, respectively. The reduction of the bosonic fields is performed by writing

$$
\begin{aligned}
& d s^{2}=g_{M N} d x^{M} d x^{N}=e^{-\frac{2}{3} \phi} g_{m n} d x^{m} d x^{n}+e^{\frac{4}{3} \phi}\left(d x^{10}-\mathcal{A}_{m} d x^{m}\right)^{2}, \\
& \mathcal{A}_{M N P}=\mathcal{A}_{m n p}, \mathcal{A}_{M N 10}=\mathcal{B}_{m n}, \text { with } M, N, P=0, \ldots, 9 .
\end{aligned}
$$

\footnotetext{
${ }^{7}$ The sign of the third term in (2.17) depends on how we distribute the constituent M-branes. We will always make the minus sign choices.
} 
Unless stated capital letters range from 0 to 10 and in the lower case from 0 to 9. The rule to generate branes with KK charges is:

(iv) If there is a common string to all constituent M-branes, say along $y$, then an electric KK charge can be added by applying a boost along the $y$ direction 111

$$
-d t^{2}+d y^{2} \rightarrow-d t^{2}+d y^{2}+\frac{\alpha}{r^{8-p}}(d y \mp d t)^{2}
$$

where the $\mp$ sign choice will correspond to positive or negative KK charge, respectively. Compactifying along the $y$ direction we have a collection of branes intersecting a 0 -brane over a point. The 2 -form field strength is then given by $(p \rightarrow p-1)$

$$
* \mathcal{F}_{2}=Q\left(\epsilon_{8-p} \wedge \eta\right)
$$

where $\eta$ is the volume form of the relative transverse space of the 0-brane $\mathcal{M}_{(0)}^{p}$. The KK electric charge is defined by $Q=\frac{1}{V_{(0)}^{p}} \int_{\Sigma} * \mathcal{F}_{2}$ with $V_{(0)}^{p}$ the volume of $\mathcal{M}_{(0)}^{p}$ and $\Sigma=S^{8-p} \times \mathcal{M}_{(0)}^{p}$. This charge is given by

$$
\frac{Q}{(7-p) A_{8-p}}= \pm \alpha
$$

If the overall transverse space has dimension bigger than three, a magnetic monopole can be added by making all the harmonic functions to depend only on three of this space coordinates and performing the substitution (we start with an anisotropic $p$-brane)

$$
\begin{aligned}
d x^{i} d x_{i} \rightarrow & d y_{p+1}^{2}+\ldots+d y_{6}^{2} \\
& +H^{-1}\left(d y_{7} \pm \alpha \cos \theta d \phi\right)^{2}+H\left(d r^{2}+r^{2} d \Omega_{2}^{2}\right)
\end{aligned}
$$

where we have introduced spherical coordinates, $H=1+\frac{\alpha}{r}$ and the \pm sign choice will correspond to positive or negative KK charge, respectively. Compactifying along the $y_{7}$ direction we have a collection of branes superposed over a 6 -brane. The 2 -form field strength is then given by

$$
\mathcal{F}_{2}=P \epsilon_{2}
$$

where the KK magnetic charge is defined by $P=\int_{S^{2}} \mathcal{F}_{2}$ and is given by

$$
\frac{P}{A_{2}}= \pm \alpha
$$

There are cases where we can add both electric and magnetic KK charges and a further compactification is required. 


\subsection{Supersymmetry}

The previous rules yield supersymmetric backgrounds. The amount of unbroken supersymmetries is determined by the rule:

(v) A configuration with $n$ constituent M-branes and KK charges preserves $2^{-n}$ of the maximal supersymmetry. 8 There are exceptions to this rule. Namely, the embeddings of the four dimensional dyonic black hole with finite horizon area have $n=4$ but preserve $\frac{1}{8}$ of the maximal supersymmetry or break all supersymmetries. This happens because the conditions on the Killing spinor field due to each of the constituent branes are not independent or are incompactible, respectively.

The fact that we considered supersymmetric backgrounds means that they will satisfy a Bogolmol'nyi bound for the ADM mass [16, 28 - 31]. The ADM mass per unit of $p$-volume for an anisotropic $p$-brane in $\mathrm{D}$ dimensions described by the Einstein's frame metric

$$
d s^{2}=-A(r) d t^{2}+\sum_{k=1}^{p} B_{k}(r) d y_{k}^{2}+C(r)\left(d x_{1}^{2}+\ldots+d x_{D-p-1}^{2}\right),
$$

is given by a straightforward generalisation of the result presented in [32]

$$
\frac{M}{A_{D-2-p}}=-\frac{1}{2}\left[r^{D-2-p} \partial_{r}\left(\sum_{k=1}^{p} B_{k}(r)+(D-2-p) C(r)\right)\right]_{r \rightarrow+\infty} .
$$

We wrote this result in $\mathrm{D}$ dimensions in order to accommodate the cases with electric or magnetic (or both) KK charges. Labelling all the charges by $Q_{i}$ (electric, magnetic and $\mathrm{KK}$ in origin) and defining in the $(2 \subset 5)$-brane case an electromagnetic charge $Q_{i}$ by $Q_{i}^{2}=P_{2 \subset 5, i}^{2}+Q_{2 \subset 5, i}^{2}$, we have that the composite M-brane rules imply

$$
2 M=\sum_{i=1}^{n}\left|Q_{i}\right| \text {. }
$$

\section{New composite M-branes}

In this chapter we present the new supersymmetric solutions that follow from applying the composite M-brane rules (iii) and (iv). By making some of the charges to vanish it is possible to enlarge the overall transverse space dimension of some configurations and therefore to change the harmonic functions dependence. We will only present the cases for which $p=7$. The other cases are given in the table presented in the conclusion.

\footnotetext{
${ }^{8}$ In the cases of multi-centered solutions, a given family of parallel branes contributes only once to $\mathrm{n}$.
} 


\section{$3.1(2 \subset 5) \perp(2 \subset 5) \perp(2 \subset 5)$ brane}

The most general intersecting configuration of M-branes involving the $(2 \subset 5)$ brane is obtained by intersecting 3 of these composite M-branes. This solution is described by

$$
\begin{aligned}
& d s^{2}=\left(\prod_{i=1}^{3}\left(H_{i} \tilde{H}_{i}\right)^{\frac{1}{3}}\right)\left[-\left(H_{1} H_{2} H_{3}\right)^{-1} d t^{2}+\left(H_{1} \tilde{H}_{3}\right)^{-1} d y_{1}^{2}\right. \\
&+\left(H_{1} \tilde{H}_{2}\right)^{-1} d y_{2}^{2}+\left(H_{2} \tilde{H}_{1}\right)^{-1} d y_{3}^{2}+\left(H_{2} \tilde{H}_{3}\right)^{-1} d y_{4}^{2} \\
&\left.+\left(H_{3} \tilde{H}_{2}\right)^{-1} d y_{5}^{2}+\left(H_{3} \tilde{H}_{1}\right)^{-1} d y_{6}^{2}+\left(\tilde{H}_{1} \tilde{H}_{2} \tilde{H}_{3}\right)^{-1} d y_{7}^{2}+d x^{j} d x_{j}\right] \\
& \mathcal{F}=\sum_{i=1}^{3}\left(\mathcal{F}_{(2, i)}+\mathcal{F}_{(5, i)}-\frac{\alpha_{i} \sin 2 \zeta_{i}}{2} \frac{\tilde{H}_{i}^{-2}}{r^{2}}\left(d r \wedge \xi_{i}\right)\right),
\end{aligned}
$$

where $* \mathcal{F}_{(2, i)}=Q_{i}\left(\epsilon_{2} \wedge \eta_{i}\right), \mathcal{F}_{(5, i)}=P_{i}\left(\mu_{i} \wedge \epsilon_{2}\right), H_{i}=1+\frac{\alpha_{i}}{r}, \tilde{H}_{i}=1+\frac{\tilde{\alpha}_{i}}{r}$, $\tilde{\alpha}_{i}=\alpha_{i} \cos ^{2} \zeta_{i}$ and $j=1,2,3$. The dual operations are defined for $\tilde{\alpha}_{i}=0$. $\eta_{i}$, $\mu_{i}$ and $\xi_{i}$ are the volume forms on $\mathcal{M}_{(2, i)}^{p-2}, \mathcal{M}_{(5, i)}^{p-5}$ and $\mathcal{M}_{(5 / 2, i)}^{3}$, respectively. The electric and magnetic charges are defined as before and are given by (2.18) with $p=7$. The ADM mass per unit of 7 -volume obtained from (2.29) is

$$
2 M=\sum_{i=1}^{3} \sqrt{Q_{i}^{2}+P_{i}^{2}}
$$

This background admits the following killing spinor field

$$
\epsilon=\left(\prod_{i=1}^{3}\left(H_{i} \tilde{H}_{i}\right)^{-\frac{1}{6}}\left[\left(\tilde{H}_{i}^{\frac{1}{2}} \pm H_{i}^{\frac{1}{2}} \cos \zeta_{i}\right)^{\frac{1}{2}}+\left(\tilde{H}_{i}^{\frac{1}{2}} \mp H_{i}^{\frac{1}{2}} \cos \zeta_{i}\right)^{\frac{1}{2}} \gamma_{i}\right]\right) \epsilon_{0}
$$

where

$$
\Gamma_{012} \gamma_{1} \epsilon_{0}=\mp \epsilon_{0}, \quad \Gamma_{034} \gamma_{2} \epsilon_{0}=\mp \epsilon_{0}, \quad \Gamma_{056} \gamma_{3} \epsilon_{0}=\mp \epsilon_{0},
$$

with $\gamma_{1}=\Gamma_{012} \Gamma_{458910}, \gamma_{2}=\Gamma_{034} \Gamma_{168910}$ and $\gamma_{3}=\Gamma_{056} \Gamma_{238910}$. The upper and lower signs definitions are as in $(2.14-15)$. The conditions $(3.4)$ reduce the amount of preserved supersymmetry to $\frac{1}{8}$ of the maximal supersymmetry. We note that all the gamma matrices related with a given $(2 \subset 5)$-brane commute with all the gamma matrices related with the other $(2 \subset 5)$ branes. I

\footnotetext{
${ }^{9} \mathrm{On}$ solving equation (2.3) this fact has to be used. For example, the gamma matrices $\gamma_{1}=-\Gamma_{367}, \Gamma_{012}$ and $\Gamma_{458910}$ related with the first $(2 \subset 5)$-brane commute with all the gamma matrices related with the other branes.
} 


\subsection{Adding Kałuża-Klein charges}

We can use the composite M-brane rule (iv) to generate KK charges. The new solutions are the $(2 \subset 5) \perp 5+$ boost, $(2 \subset 5) \perp 2+K K$ monopole, $2 \perp 2 \perp 2+K K$ monopole and $(2 \subset 5)+$ boost $+K K$ monopole branes.

\subsection{1 $(2 \subset 5) \perp 5+$ boost brane}

This solution is described by

$$
\begin{aligned}
& d s^{2}=\left(H_{1} \tilde{H}_{1}\right)^{\frac{1}{3}} H_{2}^{\frac{2}{3}}\left[\left(H_{1} H_{2}\right)^{-1}\left(-d t^{2}+d y_{7}^{2}+\frac{\alpha_{3}}{r}\left(d y_{7} \mp d t\right)^{2}\right)\right. \\
&+H_{2}^{-1}\left(d y_{1}^{2}+d y_{2}^{2}\right)+\left(H_{2} \tilde{H}_{1}\right)^{-1}\left(d y_{3}^{2}+d y_{4}^{2}\right) \\
&\left.+\tilde{H}_{1}^{-1} d y_{5}^{2}+H_{1}^{-1} d y_{6}^{2}+d x^{j} d x_{j}\right] \\
& \mathcal{F}=\mathcal{F}_{(2,1)}+\left(\sum_{i=1}^{2} \mathcal{F}_{(5, i)}\right)-\frac{\alpha_{1} \sin 2 \zeta_{1}}{2} \frac{\tilde{H}_{1}^{-2}}{r^{2}}\left(d r \wedge \xi_{1}\right),
\end{aligned}
$$

where $* \mathcal{F}_{(2,1)}=Q_{1}\left(\epsilon_{2} \wedge \eta_{1}\right), \mathcal{F}_{(5, i)}=P_{i}\left(\mu_{i} \wedge \epsilon_{2}\right)$ with the dual operation defined for the metric with $\tilde{\alpha}_{1}=0$ and $j=1,2,3$.

This background is $\frac{1}{8}$ supersymmetric. The Killing spinor field is

$$
\epsilon=H_{3}^{-\frac{1}{4}} H_{2}^{-\frac{1}{12}}\left(H_{1} \tilde{H}_{1}\right)^{-\frac{1}{6}}\left[\left(\tilde{H}_{1}^{\frac{1}{2}} \pm H_{1}^{\frac{1}{2}} \cos \zeta_{1}\right)^{\frac{1}{2}}+\left(\tilde{H}_{1}^{\frac{1}{2}} \mp H_{1}^{\frac{1}{2}} \cos \zeta_{1}\right)^{\frac{1}{2}} \gamma\right] \epsilon_{0}
$$

where

$$
\Gamma_{067} \gamma \epsilon_{0}=\mp \epsilon_{0}, \quad \Gamma_{568910} \epsilon_{0}=\mp \epsilon_{0}, \quad \Gamma_{07} \epsilon_{0}=\mp \epsilon_{0},
$$

with $\gamma=\Gamma_{067} \Gamma_{128910}$. The upper and lower sign choice in the last condition in (3.7) corresponds to positive or negative KK charge, respectively.

Dimensional reduction along $y_{7}$ yields the $(1 \subset 4) \perp 4 \perp 0$ brane of type IIA superstring theory

$$
\begin{aligned}
& d s_{10}^{2}=\left(\tilde{H}_{1} H_{2} H_{3}\right)^{\frac{1}{2}}\left[-\left(H_{1} H_{2} H_{3}\right)^{-1} d t^{2}+H_{2}^{-1}\left(d y_{1}^{2}+d y_{2}^{2}\right)\right. \\
&\left.+\left(H_{2} \tilde{H}_{1}\right)^{-1}\left(d y_{3}^{2}+d y_{4}^{2}\right)+\tilde{H}_{1}^{-1} d y_{5}^{2}+H_{1}^{-1} d y_{6}^{2}+d x^{j} d x_{j}\right], \\
& e^{2 \phi}= H_{1}^{-1} \tilde{H}_{1}^{\frac{1}{2}} H_{2}^{-\frac{1}{2}} H_{3}^{\frac{3}{2}}, \quad * \mathcal{F}_{2}=Q_{3}\left(\epsilon_{2} \wedge \eta_{3}\right), \quad * \mathcal{H}=-Q_{1} e^{2 \phi}\left(\epsilon_{2} \wedge \eta_{1}\right), \\
& \mathcal{F}_{4}=\left(\sum_{i=1}^{2} P_{i}\left(\mu_{i} \wedge \epsilon_{2}\right)\right)-\frac{\alpha_{1} \sin 2 \zeta_{1}}{2} \frac{\tilde{H}_{1}^{-2}}{r^{2}}\left(d r \wedge \xi_{1}\right),
\end{aligned}
$$


where $\mu_{i}, \eta_{1}, \eta_{3}$ and $\xi_{1}$ are the volume forms on the spaces $\mathcal{M}_{(4, i)}^{2}, \mathcal{M}_{(1,1)}^{5}, \mathcal{M}_{(0,3)}^{6}$ and $\mathcal{M}_{(4 / 1,1)}^{3}$, respectively. Note that according to (2.20) dimensional reduction

of $\mathcal{F}_{(2,1)}$ gives $\mathcal{H}_{t r y_{6}}=\frac{Q_{1}}{A_{2}} \frac{H_{1}^{-2}}{r^{2}}$. Following the definitions in footnote 3 the field strength $\mathcal{H}$ can be written as in (3.8).

\subsection{2 $(2 \subset 5) \perp 2+K K$ monopole brane}

This solution is given by

$$
\begin{aligned}
& d s^{2}=\left(H_{1} \tilde{H}_{1} H_{2}\right)^{\frac{1}{3}}\left[-\left(H_{1} H_{2}\right)^{-1} d t^{2}+H_{1}^{-1}\left(d y_{1}^{2}+d y_{2}^{2}\right)\right. \\
&+\tilde{H}_{1}^{-1}\left(d y_{3}^{2}+d y_{4}^{2}\right)+\left(\tilde{H}_{1} H_{2}\right)^{-1} d y_{5}^{2}+H_{2}^{-1} d y_{6}^{2} \\
&\left.+H_{3}^{-1}\left(d y_{7} \pm \alpha_{3} \cos \theta d \phi\right)^{2}+H_{3}\left(d r^{2}+r^{2} d \Omega_{2}^{2}\right)\right] \\
& \mathcal{F}=\left(\sum_{i=1}^{2} \mathcal{F}_{(2, i)}\right)+\mathcal{F}_{(5,1)}-\frac{\alpha_{1} \sin 2 \zeta_{1}}{2} \frac{\tilde{H}_{1}^{-2}}{r^{2}}\left(d r \wedge \xi_{1}\right),
\end{aligned}
$$

where $* \mathcal{F}_{(2, i)}=Q_{i}\left(\epsilon_{2} \wedge \eta_{i}\right)$ and $\mathcal{F}_{(5,1)}=P_{1}\left(\mu_{1} \wedge \epsilon_{2}\right)$.

This background is $\frac{1}{8}$ supersymmetric. The Killing spinor field is

$$
\epsilon=\left(H_{1} \tilde{H}_{1} H_{2}\right)^{-\frac{1}{6}}\left[\left(\tilde{H}_{1}^{\frac{1}{2}} \pm H_{1}^{\frac{1}{2}} \cos \zeta_{1}\right)^{\frac{1}{2}}+\left(\tilde{H}_{1}^{\frac{1}{2}} \mp H_{1}^{\frac{1}{2}} \cos \zeta_{1}\right)^{\frac{1}{2}} \gamma\right] \epsilon_{0},
$$

where

$$
\Gamma_{012} \gamma \epsilon_{0}=\mp \epsilon_{0}, \quad \Gamma_{056} \epsilon_{0}=\mp \epsilon_{0}, \quad \Gamma_{78910} \epsilon_{0}= \pm \epsilon_{0},
$$

with $\gamma=\Gamma_{012} \Gamma_{678910}$. The upper and lower sign choice in the last condition in (3.11) corresponds to positive or negative KK charge, respectively.

Dimensional reduction along $y_{7}$ yields the $((2 \subset 5) \perp 2) \| 6$ brane of type IIA superstring theory

$$
\begin{aligned}
& d s_{10}^{2}=\left(H_{1} \tilde{H}_{1} H_{2} H_{3}\right)^{\frac{1}{2}}\left[-\left(H_{1} H_{2} H_{3}\right)^{-1} d t^{2}\right. \\
&+\left(H_{1} H_{3}\right)^{-1}\left(d y_{1}^{2}+d y_{2}^{2}\right)+\left(\tilde{H}_{1} H_{3}\right)^{-1}\left(d y_{3}^{2}+d y_{4}^{2}\right) \\
&\left.+\left(\tilde{H}_{1} H_{2} H_{3}\right)^{-1} d y_{5}^{2}+\left(H_{2} H_{3}\right)^{-1} d y_{6}^{2}+d x^{j} d x_{j}\right] \\
& e^{2 \phi}=\left(H_{1} \tilde{H}_{1} H_{2}\right)^{\frac{1}{2}} H_{3}^{-\frac{3}{2}}, \quad \mathcal{H}=P_{1}\left(\mu_{1} \wedge \epsilon_{2}\right), \quad \mathcal{F}_{2}=P_{3} \epsilon_{2} \\
& \mathcal{F}_{4}=\left(\sum_{i=1}^{2} \mathcal{F}_{(2, i)}\right)-\frac{\alpha_{1} \sin 2 \zeta_{1}}{2} \frac{\tilde{H}_{1}^{-2}}{r^{2}}\left(d r \wedge \xi_{1}\right) .
\end{aligned}
$$




\subsection{3 $2 \perp 2 \perp 2+K K$ monopole brane}

There is a new configuration not involving the $(2 \subset 5)$-brane that can be obtained by adding a KK monopole to the $2 \perp 2 \perp 2$ brane. It provides a new embedding of the extreme four dimensional dyonic black hole with finite horizon area. This solution is described by

$$
\begin{aligned}
& d s^{2}=\left(H_{1} H_{2} H_{3}\right)^{\frac{1}{3}}\left[-\left(H_{1} H_{2} H_{3}\right)^{-1} d t^{2}+H_{1}^{-1}\left(d y_{1}^{2}+d y_{2}^{2}\right)\right. \\
&+H_{2}^{-1}\left(d y_{3}^{2}+d y_{4}^{2}\right)+H_{3}^{-1}\left(d y_{5}^{2}+d y_{6}^{2}\right) \\
&\left.\quad+H_{4}^{-1}\left(d y_{7} \pm \alpha_{4} \cos \theta d \phi\right)^{2}+H_{4}\left(d r^{2}+r^{2} d \Omega_{2}^{2}\right)\right], \\
& * \mathcal{F}=\sum_{i=1}^{3} Q_{i}\left(\epsilon_{2} \wedge \eta_{i}\right) .
\end{aligned}
$$

This background admits the killing spinor field

$$
\epsilon=\left(H_{1} H_{2} H_{3}\right)^{-\frac{1}{6}} \epsilon_{0},
$$

where

$$
\Gamma_{012} \epsilon_{0}=\mp \epsilon_{0}, \quad \Gamma_{034} \epsilon_{0}=\mp \epsilon_{0}, \quad \Gamma_{056} \epsilon_{0}=\mp \epsilon_{0} .
$$

The supersymmetry breaking condition due to the KK monopole is $\Gamma_{78910} \epsilon_{0}=$ $\pm \epsilon_{0}$. Consider first the upper sign choice, i.e. positive KK charge, this condition can be obtained from (3.15) if there are three positively or one positively and two negatively charged 2-branes. If this is the case the solution preserves $\frac{1}{8}$ of the maximal supersymmetry. Otherwise the KK monopole supersymmetry breaking condition is incompactible with (3.15) and the solution breaks all supersymmetries. Similar comments apply for negative KK charge. An analogous situation occurs for the other embeddings of the extreme four dimensional dyonic black hole with finite horizon area.

Compactifying the solution (3.13) along the $y_{7}$ direction we obtain the $(2 \perp$ $2 \perp 2) \| 6$ brane of type IIA superstring theory

$$
\begin{gathered}
d s_{10}^{2}=\left(H_{1} H_{2} H_{3} H_{4}\right)^{\frac{1}{2}}\left[-\left(H_{1} H_{2} H_{3} H_{4}\right)^{-1} d t^{2}+\left(H_{1} H_{4}\right)^{-1}\left(d y_{1}^{2}+d y_{2}^{2}\right)\right. \\
\left.+\left(H_{2} H_{4}\right)^{-1}\left(d y_{3}^{2}+d y_{4}^{2}\right)+\left(H_{3} H_{4}\right)^{-1}\left(d y_{5}^{2}+d y_{6}^{2}\right)+d x^{j} d x_{j}\right] \\
e^{2 \phi}=\left(H_{1} H_{2} H_{3}\right)^{\frac{1}{2}} H_{4}^{-\frac{3}{2}}, \quad * \mathcal{F}_{4}=\sum_{i=1}^{3} Q_{i}\left(\epsilon_{2} \wedge \eta_{i}\right), \quad \mathcal{F}_{2}=P_{4} \epsilon_{2} .
\end{gathered}
$$

Applying T-duality along one direction of each 2-brane we get the $3 \perp 3 \perp 3 \perp 3$ brane of type IIB superstring theory [12]. 


\subsection{4 $2 \subset 5+$ boost $+K K$ monopole brane}

It is straightforward by applying the composite M-brane rule (iv) to obtain the brane solution $2 \subset 5+$ boost $+K K$ monopole. It is given by

$$
\begin{gathered}
d s^{2}=\left(H_{1} \tilde{H}_{1}\right)^{\frac{1}{3}}\left[H_{1}^{-1}\left(-d t^{2}+d y_{1}^{2}+\frac{\alpha_{2}}{r}\left(d y_{1} \mp d t\right)^{2}+d y_{2}^{2}\right)\right. \\
+\tilde{H}_{1}^{-1}\left(d y_{3}^{2}+d y_{4}^{2}+d y_{5}^{2}\right)+d y_{6}^{2} \\
\left.\quad+H_{3}^{-1}\left(d y_{7} \pm \alpha_{3} \cos \theta d \phi\right)^{2}+H_{3}\left(d r^{2}+r^{2} d \Omega_{2}^{2}\right)\right], \\
\mathcal{F}=\mathcal{F}_{(2,1)}+\mathcal{F}_{(5,1)}-\frac{\alpha_{1} \sin 2 \zeta_{1}}{2} \frac{\tilde{H}_{1}^{-2}}{r^{2}}\left(d r \wedge \xi_{1}\right),
\end{gathered}
$$

where $* \mathcal{F}_{(2,1)}=Q_{1}\left(\epsilon_{2} \wedge \eta_{1}\right)$ and $\mathcal{F}_{(5,1)}=P_{1}\left(\mu_{1} \wedge \epsilon_{2}\right)$.

This background preserves $\frac{1}{8}$ of the maximal supersymmetry. The corresponding Killing spinor field is

$$
\epsilon=H_{2}^{-\frac{1}{4}}\left(H_{1} \tilde{H}_{1}\right)^{-\frac{1}{6}}\left[\left(\tilde{H}_{1}^{\frac{1}{2}} \pm H_{1}^{\frac{1}{2}} \cos \zeta_{1}\right)^{\frac{1}{2}}+\left(\tilde{H}_{1}^{\frac{1}{2}} \mp H_{1}^{\frac{1}{2}} \cos \zeta_{1}\right)^{\frac{1}{2}} \gamma\right] \epsilon_{0}
$$

where

$$
\Gamma_{012} \gamma \epsilon_{0}=\mp \epsilon_{0}, \quad \Gamma_{01} \epsilon_{0}=\mp \epsilon_{0}, \quad \Gamma_{78910} \epsilon_{0}= \pm \epsilon_{0},
$$

with $\gamma=\Gamma_{012} \Gamma_{678910}$.

Dimensional reduction along the monopole direction $y_{7}$ yields the $((2 \subset 5)+$ boost) $\| 6$ brane solution of type IIA superstring theory. This solution is described by

$$
\begin{aligned}
& d s_{10}^{2}=\left(H_{1} \tilde{H}_{1} H_{3}\right)^{\frac{1}{2}}\left[\left(H_{1} H_{3}\right)^{-1}\left(-d t^{2}+d y_{1}^{2}+\frac{\alpha_{2}}{r}\left(d t \mp d y_{1}\right)^{2}+d y_{2}^{2}\right)\right. \\
&\left.+\left(\tilde{H}_{1} H_{3}\right)^{-1}\left(d y_{3}^{2}+d y_{4}^{2}+d y_{5}^{2}\right)+H_{3}^{-1} d y_{6}^{2}+d x^{j} d x_{j}\right], \\
& e^{2 \phi}=\left(H_{1} \tilde{H}_{1}\right)^{\frac{1}{2}} H_{3}^{-\frac{3}{2}}, \quad \mathcal{H}=P_{1}\left(\mu_{1} \wedge \epsilon_{2}\right), \quad \mathcal{F}_{2}=P_{3} \epsilon_{2}, \\
& \mathcal{F}_{4}=\mathcal{F}_{(2,1)}-\frac{\alpha_{1} \sin 2 \zeta_{1}}{2} \frac{\tilde{H}_{1}^{-2}}{r^{2}}\left(d r \wedge \xi_{1}\right) .
\end{aligned}
$$

A further reduction yields a $((1 \subset 4) \perp 0) \| 5$ brane solution of nine dimensional type IIA superstring theory. We could have reduced the solution (3.17) along the boost direction to obtain the $(1 \subset 4) \perp 0+K K$ monopole brane of type IIA superstring theory. A further reduction along the monopole direction yields a $((1 \subset 4) \perp 0) \| 5$ brane solution of the corresponding nine dimensional theory. Note that this brane solution is not the same as the previous one as the basic branes charges arise from different fields strength. 
Table 1: Composite M-brane scan

\begin{tabular}{|c|c|c|c|c|}
\hline & Composite M-brane & SUSY & $p$ & $D=11-p$ \\
\hline \hline$N=4$ & $2 \perp 2 \perp 5 \perp 5$ & $1 / 8,0$ & 7 & $4 \dagger$ \\
\hline$N=3$ & $(2 \subset 5) \perp(2 \subset 5) \perp(2 \subset 5)$ & $1 / 8$ & 7 & 4 \\
& $2 \perp 2 \perp 2$ & $1 / 8$ & 6 & $5 \ddagger$ \\
\hline$N=3$ & $5 \perp 5 \perp 5+$ boost & $1 / 8,0$ & 7 & $4 \dagger$ \\
$+\mathrm{KK}$ charges & $2 \perp 2 \perp 2+K K$ monopole & $1 / 8,0$ & 7 & $4 \dagger$ \\
\hline$N=2$ & $(2 \subset 5) \perp(2 \subset 5)$ & $1 / 4$ & 7 & 4 \\
& $(2 \subset 5) \perp 2$ & $1 / 4$ & 6 & 5 \\
& $2 \perp 2$ & $1 / 4$ & 4 & 7 \\
\hline$N=2$ & $(2 \subset 5) \perp 5+$ boost & $1 / 8$ & 7 & 4 \\
$+\mathrm{KK}$ charges & $(2 \subset 5) \perp 2+K K$ monopole & $1 / 8$ & 7 & 4 \\
& $2 \perp 5+$ boost $+K K$ monopole & $1 / 8,0$ & 7 & $4 \dagger$ \\
& $2 \perp 5+$ boost & $1 / 8$ & 6 & $5 \ddagger$ \\
\hline$N=1$ & $2 \subset 5$ & $1 / 2$ & 5 & 6 \\
& 2 & $1 / 2$ & 2 & 9 \\
\hline$N=1$ & $(2 \subset 5)+$ boost + KK monopole & $1 / 8$ & 7 & 4 \\
$+\mathrm{KK}$ charges & $(2 \subset 5)+$ boost & $1 / 4$ & 5 & 6 \\
& $2+$ boost & $1 / 4$ & 2 & 9 \\
\hline KK charges & boost $+K K$ monopole & $1 / 4$ & - & 4 \\
& boost & $1 / 2$ & - & 10 \\
\hline
\end{tabular}

\section{Conclusion}

We have found new intersecting M-brane configurations that follow from the composite M-brane rules stated in section 2. These new supersymmetric solutions are built by intersecting the 2, 5 and $2 \subset 5$ branes and, in some cases, by adding KK charges. In table 1 we present the composite M-brane scan resulting from intersecting $N$ of the 2,5 and $2 \subset 5$ branes. These solutions may be seen as anisotropic $p$-branes. The corresponding amount of preserved supersymmetry is also shown. There are configurations that are not presented but can be obtained from other configurations in the table, e.g. the basic 5 -brane can be obtain from the $(2 \subset 5)$-brane. By allowing the harmonic functions to depend on just some coordinates of the overall transverse space, all configurations with $p<7$ can be made a r-brane with $p<r \leq 7$. Dimensional reduction along the branes spatial directions yields black hole solutions in $D=11-p$ dimensions (for $p<7$ we can have $4 \leq D \leq 11-p$ ). The solutions marked with $\dagger$ and $\ddagger$ are respectively, embeddings of the four [23-25] and five [33] dimensional dyonic black holes with finite horizon area. Note that the latter always yields the former by superposing a KK monopole. 
It seems interesting to study the supersymmetric brane solutions of string theories that arrive by dimensional reduction of these M-branes and further duality transformations $[11,13,17-19]$. This could provide a set of composite rules derived from the rather simple rules in M-theory.

All solutions that have been considered can be generalised to the corresponding black configurations, providing an opportunity of doing thermodynamics by using the corresponding M-theory rules [34-36].

\section{Acknowledgements}

The author is grateful to M.J.Perry for introducing him into the M-theory subject and acknowledges the financial support of JNICT (Portugal) under program PRAXIS XXI. 


\section{References}

[1] P.K.Townsend, Phys. Lett. B350 (1995) 184.

[2] E.Witten, Nucl. Phys. B443 (1995) 85.

[3] M.J.Duff and K.S.Stelle, Phys. Lett. B253 (1991) 113.

[4] R.Güven, Phys. Lett. B276 (1992) 49.

[5] E.Bergshoeff, E.Sezgin and P.K.Townsend, Phys. Lett. B189 (1987) 75; Ann. Phys. 185 (1988) 330.

[6] M.J.Duff, P.S.Howe, T.Inami and K.S.Stelle, Phys. Lett. B191 (1987).

[7] P.Hořava and E.Witten, Nucl. Phys. B460 (1996) 506; Nucl. Phys. B475 (1996) 94.

[8] J.H.Schwarz, Phys. Lett. B360 (1995) 13, Erratum B364 (1995) 252; Superstring dualities, hep-th/9509148; Phys. Lett. B367 (1996) 97.

[9] P.S.Aspinwall, Some Relationships between dualities in string theory, hepth/9508154.

[10] G.Papadopoulos and P.K.Townsend, Phys. Lett. B380 (1996) 273.

[11] A.A.Tseytlin, Nucl. Phys. B475 (1996) 149.

[12] I.R.Klebanov and A.A.Tseytlin, Nucl. Phys. B475 (1996) 179.

[13] J.P.Gauntlett, D.A.Kastor and J.Traschen, Overlapping Branes in M-theory, hep-th/9604179.

[14] D.Gross and M.J.Perry, Nucl. Phys. B226 (1983) 29.

[15] R.Sorkin, Phys. Rev. Lett. 51 (1983) 87.

[16] J.M.Izquierdo, N.D.Lambert, G.Papadopoulos and P.K.Townsend, Nucl. Phys. B460 (1996) 560.

[17] H.Lü and C.N.Pope, Nucl. Phys. B465 (1996) 127; Multi-scalar p-brane solitons, hep-th/9512153.

[18] N.Khviengia, Z.Khviengia, H.Lü and C.N.Pope, Intersecting M-branes and Bound States, hep-th/9605077.

[19] K.Behrndt, E.Bergshoeff and B.Janssen, Intersecting D-Branes in Ten and Six Dimensions, hep-th/9604168. 
[20] I.R.Klebanov and A.A.Tseytlin, Nucl. Phys. B475 (1996) 164.

[21] A.Hanany and I.R.Klebanov, On Tensionless Strings in 3+1 Dimensions, hep-th/9606136.

[22] I.R.Klebanov and A.A.Tseytlin, Near-Extremal Black Hole Entropy and Fluctuating 3-Branes, hep-th/9607107.

[23] M.Cvetič and D.Youm, Phys. Rev. D53 (1996) 584.

[24] M.Cvetič and A.A.Tseytlin, Phys. Lett. B366 (1996)95.

[25] M.Cvetič and A.A.Tseytlin, Phys. Rev. D53 (1996) 5619.

[26] A.Strominger, Phys. Lett. B383 (1996) 44.

[27] P.K.Townsend, Phys. Lett. B373 (1996) 68.

[28] E.Witten, Commun. Math. Phys. 80 (1981) 381.

[29] G.W.Gibbons and C.M. Hull, Phys. Lett. 109B (1982) 190.

[30] A.Dabholkar, G.W.Gibbons, J.A.Harvey and F.R.Ruiz, Nucl. Phys. B340 (1990) 33.

[31] G.W.Gibbons, G.T.Horowitz and P.K.Townsend, Class. Quantum Grav. 12 (1995) 297.

[32] J.X.Lü, Phys. Lett. B313 (1993) 29.

[33] A.A.Tseytlin, Mod. Phys. Lett. A11 (1996) 689.

[34] M.J.Duff, H.Lü and C.N.Pope, Phys. Lett. B382 (1996) 73.

[35] M.Cvetič and A.A.Tseytlin, Non-Extreme Black Holes from Non-Extreme Intersecting M-branes, hep-th 9606033 .

[36] M.S.Costa, Black composite M-branes, in preparation. 